\title{
El Moltó Tarragoní, una raza antigua extinguida
}

\author{
Álvarez, S.
}

\section{Palabras ClaVE ADICIONALES}

Peligro de extinción.

\section{ADDITIONAL KEYWORDS}

Endangered breed.

INFORMACIÓN

Cronología del artículo.

Recibido/Received: 2.3.2015

Aceptado/Accepted: 14.7.2015

On-line: 16.11.2015

Correspondencia a los autores/Contact e-mail:

santalba@hotmail.com

\section{RESUMEN}

Se describe una raza ovina antigua de la zona central de Cataluña, el Moltó Tarragoní (carnero tarraconense). Se detallan sus posibles relaciones con otras razas ovinas próximas y se analizan las causas que contribuyeron a su temprana desaparición.

\section{Tarragona Ram, an ancient extinct breed}

SUMMARY

An old sheep breed (Moltó Tarragoní) in the central region of Catalonia is described; his possible relationships with other nearby sheep breeds are detailed and are analyzed the causes that contributed to its early extinction.

\section{INTRODUCCIÓN}

En el último medio siglo se ha incrementado el número de razas domésticas de ganado que se encuentran en peligro de extinción y algunas de ellas ya han desaparecido. En España más del 80\% de las razas autóctonas incluidas en el Catálogo oficial de razas de ganado de España se encuentran en peligro de extinción.

En este sentido, Hall et al. (1993) estiman que el número de razas autóctonas extinguidas durante el último siglo supera las 600, representando un 16\% del total mundial. Según estos autores la extinción de razas ganaderas ha sido más acusada en Europa y en la antigua Unión Soviética. Actualmente este proceso de extinción también se ha extendido a otros países en vías de desarrollo.

La FAO (2010) ha elaborado un inventario de las razas ganaderas de la tierra en el que se incluyen las principales amenazas que se ciernen sobre ellas. Según reconoce esta misma organización en sus informes, resulta complejo mantener un registro de todo el patrimonio ganadero mundial y más si cabe del que ya se ha extinguido ya que la calidad de la información contenida en su base de datos es discutible ya que hay poca información bibliográfica que permita documentar la desaparición del patrimonio ganadero mundial y por esta razón no es posible establecer con certeza las tendencias pasadas de los recursos ganaderos.

Actualmente la FAO ha publicado la Lista mundial de vigilancia para la diversidad de los animales domésticos (Scherf, 2000) y gestiona el Banco de datos global de los recursos genéticos de los animales domésticos en los que se almacena la información conocida sobre los recursos zoogenéticos mundiales. Esta base de datos incluye un registro de las razas extinguidas conocidas en el que se añade, cuando es posible, la causa de su desaparición.

Algunos países también mantienen registros paralelos de su biodiversidad ganadera. En Estados Unidos de América, la American Livestock Breeds Conservacy (ALBC) revisa periódicamente el estado de conservación de sus razas ganaderas autóctonas. En la misma dirección trabaja la Rare Breeds Canada (RBC) que evalúa, estudia y conserva sus razas más vulnerables.

En el caso concreto de España, la bibliografía permite constatar la extinción de algunas razas ganaderas. Actualmente el banco de datos de la FAO incluye 23 razas ganaderas extinguidas.

El estudio de estas razas ya extinguidas, resulta interesante y necesario desde un punto de vista histórico, cultural y etnográfico. Comprender y analizar las causas que las llevaron a desaparecer puede ayudar a conservar otras razas ganaderas amenazadas o vulnerables.

En este trabajo se exponen algunos aspectos de una raza ovina autóctona ya desaparecida conocida como Moltó Tarragoní. Se trata de una población que estudió el doctor Emili Giralt i Raventós, antiguo director del Centre d'Estudis d'Història Rural de Cataluña. Lamentablemente su inesperada desaparición precipitó que sus conclusiones, no viesen la luz. Desde aquí queremos agradecer a sus hijas, Mireia y Olga Giralt Esteve, su 
predisposición y colaboración al facilitar la consulta del archivo del doctor Giralt, que incluye numerosos documentos, la mayoría de finales del siglo XIX, que se han revelado como una interesante fuente de información y conocimiento de esta raza.

\section{ETIMOLOGÍA Y SINONIMIA}

La denominación habitual que tradicionalmente ha recibido esta antigua raza en lengua catalana ha sido Moltó Tarragoní, que podría traducirse como carnero tarraconense. Este apelativo aparece ya documentado durante el siglo XVII, compaginándose simultáneamente con otras denominaciones como Moltó Negre o Moltó de la Terra (Bosch et al., 1992).

La comarca de la Segarra (Lérida) fue la cuna de esta antigua raza ganadera, motivo que explica la aparición de otras denominaciones locales menos conocidas como raza Segarrenca (Rovira, 1884). Este hecho ha propiciado que algunos autores hayan confundido e incluso diferenciado el Moltó Tarragoní y la raza Segarrenca como dos poblaciones distintas.

\section{DESCRIPCIÓN MORFOLÓGICA Y PROTOTIPO RACIAL}

El Moltó Tarragoní aparece descrito en varios documentos fechados entre los siglos XVII y XIX. Se trataba de una raza de reconocido prestigio carnicero tanto en su territorio tradicional de explotación como fuera de él, alcanzando su fama a toda Cataluña.

A través de la descripción realizada por Virgili (1892), ingeniero del servicio agronómico provincial de la provincia de Tarragona, hoy nos podemos hacer una idea precisa de las características morfológicas de esta población.

Se trataba de un ganado de gran alzada, de mayor tamaño que el resto de agrupaciones raciales vecinas. Su cabeza era grande y de perfil marcadamente convexo, estando desprovista de lana. Sus ojos eran grandes y vivos, sus orejas lisas y pequeñas de cuello largo y poco grueso. Su tronco era alto, alargado, ancho $\mathrm{y}$ redondeado de cuarto trasero. Su vientre desprovisto de lana, lo mismo que las extremidades que eran largas y delgadas vistas desde frente, pero muy anchas vistas de perfil.

Un elemento diferenciador de la raza era su cornamenta. Estaba presente en la totalidad de los ejemplares y se caracterizaba por ser gruesa, baja y pegada a la cabeza en forma de media luna y poco enrollada en espiral. La existencia en los rebaños de ejemplares mochos denotaba cruzamiento y falta de pureza.

El color dominante y típico de la raza era el negro, aunque ya eran muy frecuentes, a finales del siglo XIX, los rebaños integrados por animales de color blanco, debido al mestizaje que venía impuesto por los nuevos cánones productivos de la época.

Los sementales conservaban un carácter de bravura que los hacía especialmente feroces en las peleas de monta y difíciles de manejar cuando alcanzaban cierta edad.

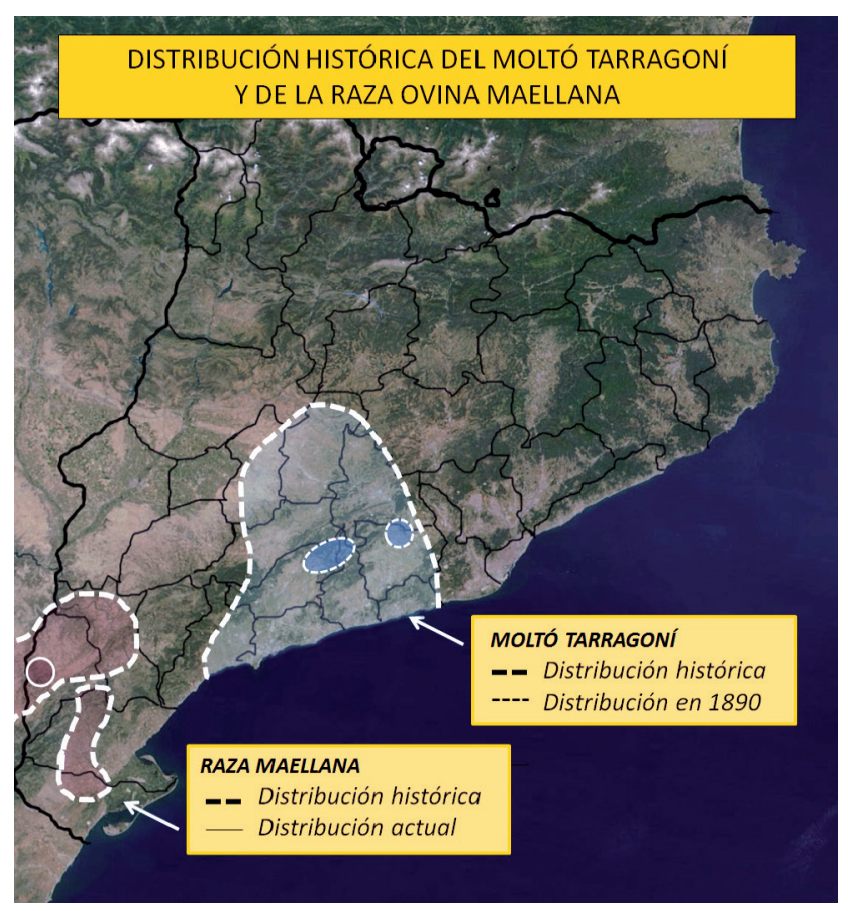

Figura 1. Distribución histórica de la raza ovina Maellana y del Moltó Tarragoní en Cataluña. Elaboración propia (Historical range of the Maellana sheep breed and the Moltó Tarragoní ovine breed in Catalonia. Authors).

\section{IMPORTANCIA Y EXTENSIÓN - ZONA DE ORIGEN}

El ámbito territorial del Moltó Tarragoní no se circunscribía únicamente a la provincia de Tarragona ya que también se documenta su presencia en algunos municipios limítrofes de las provincias de Barcelona y Lleida.

Virgili (1892), define y delimita este territorio con precisión. Por el sur limitaba con el mar mediterráneo, una frontera natural que hoy coincidiría aproximadamente con la franja litoral que ocupa la Costa Dorada, por el norte penetraba en la comarca de la Segarra hasta llegar a Cervera y se internaba también en la provincia de Barcelona a la altura de la localidad de Pujalt, alcanzando también las localidades de Calaf, Igualada y los llanos del Penedés (figura 1).

El mismo autor señala que, a pesar de su denominación la raza no estaba presente en todos los partidos judiciales de la provincia de Tarragona, faltando de los de Gandesa, Tortosa y Falset, en los que se podían encontrar otras dos agrupaciones raciales distintas, una de naturaleza serrana y otra, en la zona de influencia de Falset (oveja Maellana).

El territorio del Moltó Tarragoní se vio reducido de manera muy importante durante la segunda mitad del siglo XIX. Hacia 1890 prácticamente sólo podía encontrarse en las inmediaciones de la localidad de Sant Sadurní d'Anoia (Alt Penedés). En el año 1891 solamente restaban algunos ejemplares mestizos en las montañas de Montagut y Selma.

Durante las primeras décadas del siglo XX, continuó la importación de ganado aragonés y castellano, y el territorio del Moltó Tarragoní fue incorporando progresivamente este nuevo ganado que propició entre 


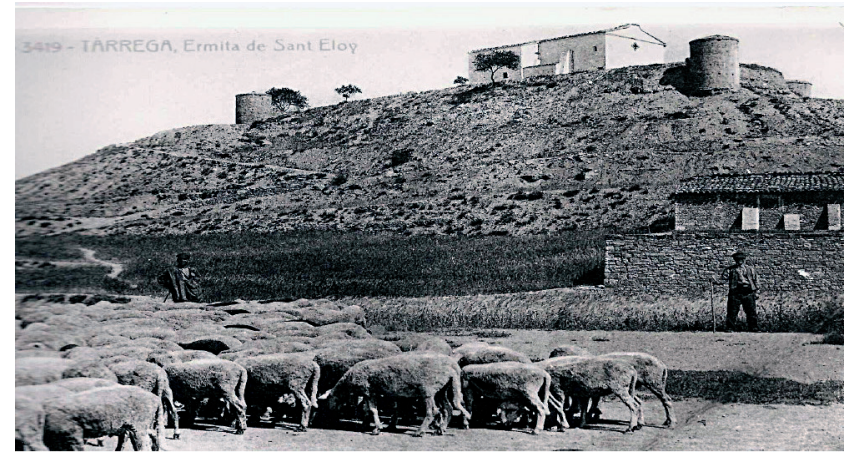

Figura 2. A principios del siglo XX, el Moltó Tarragoní había desaparecido completamente de sus territorios históricos (In the early twentieth century, Moltó Tarragoní ovine breed had completely disappeared from their historical territories).

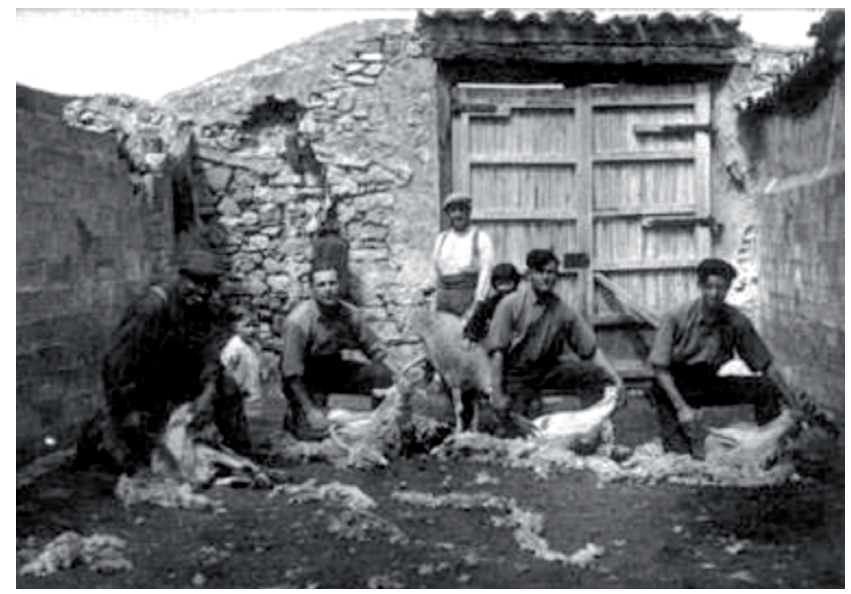

Figura 3. Cuadrilla de esquiladores en la comarca de la Segarra, cuna del Moltó Tarragoní. Archivo Claudi Gómez Grau (Shearers in the region of the Segarra, birthplace of Moltó Tarragoni ovine breed).

otras cosas: una disminución de su talla, el cambió de coloración de su vellón, un incremento progresivo de su lana y la pérdida de su cornamenta (figura 2). Pocas décadas después, hacia 1930, Rosell i Vila describe el ganado ovino que observa en la comarca de la Segarra y por aquel entonces ya se encontraba bastante degenerado aunque presentaba tanto pelo como lana, característica esta última probablemente heredada del antiguo Moltó Tarragoní (figura 3).

\section{EL MOLTÓ TARRAGONÍ Y LA OVEJA MAELLANA}

Gracias a la agudeza descriptiva de Virgili (1892), sabemos que el Moltó Tarragoní era muy similar a la oveja Maellana (figura 4). A este autor también se le debe el mérito de haber descrito y documentado la raza Maellana por vez primera:

El lanar que posee el Sr. Serrate, inteligente ganadero de Gandesa, tiene la cabeza muy acarnerada y desnuda, espina dorsal larga, es ancho y redondeado del cuarto trasero, alto y ancho de patas, cuello algo largo y con pelo de cabra. Recuerda al Moltó Tarragoní, pero es blanco y mocho como todo el del país. [...]. Este ganado es preferido en toda la comarca y su poseedor tiene especial cuidado en la elección de los moruecos que adquiere de otros rebaños, porque opina que eligiéndolos del propio para cubrir las ovejas hermanas se obtienen crías enfermas o locas.

La raza Maellana, hasta mediados del siglo XX se extendía por las zonas llanas de las Tierras del Ebro catalanas, incluyendo localidades como Batea, Mora d'Ebre, Bot, Ascó, Gandesa y descendiendo río abajo alcanzaba la localidad de Tortosa. Su territorio se ha visto reducido de manera muy importante durante las últimas décadas aunque en Cataluña todavía se conserva un último rebaño en la localidad de Arnes que está integrado dentro del libro genealógico de la raza.

La oveja Maellana hoy ocupa un territorio más reducido que el que ocupó en el pasado que en cierto modo se sostiene gracias a las ayudas europeas y que hoy coincide a grandes rasgos con el triángulo que definen las localidades de Caspe, Alcañiz y Gandesa que tiene como epicentro a la localidad de Maella.

El Moltó Tarragoní y la oveja Maellana tenían varios elementos en común, además de presentar un gran parecido morfológico ocupaban territorios muy próximos. En este sentido, dentro de las explotaciones ganaderas de raza Maellana, aparecen todavía ejemplares de capa negra que el estándar oficial de la raza impide inscribir en su libro genealógico (figura 5). Estos ejemplares negros serían hoy muy similares al antiguo Moltó Tarragoní.

Entre los ganaderos antiguos de la Terra Alta existía la antigua superstición de que estas ovejas negras auguraban buena suerte y tenían la capacidad de alejar a los rayos y males del rebaño. A estas ovejas, de coloración totalmente negra, se les denominaba centelleres y todavía hoy los ganaderos de Arnes coinciden en señalar que no se les debe infligir ningún daño ni hacer sangre, ya que de otro modo pierden estas facultades.

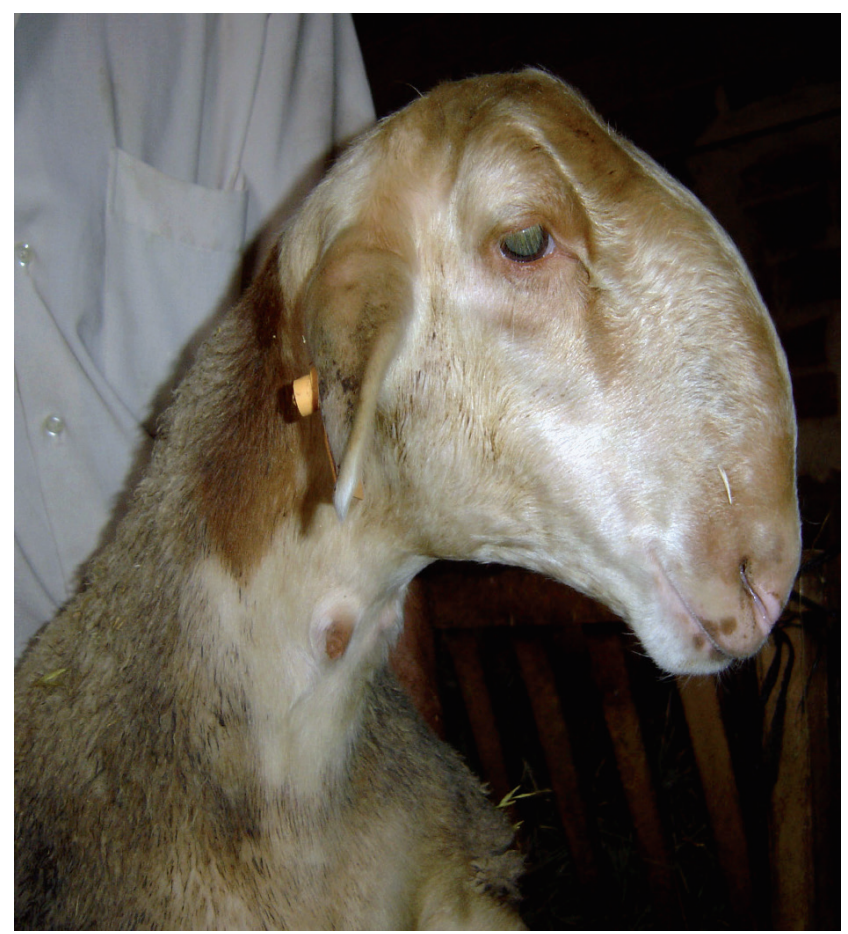

Figura 4. Morueco de raza Maellana procedente de la localidad tarraconense de Arnes (Maellana ram from Arnes (Tarragona)). 


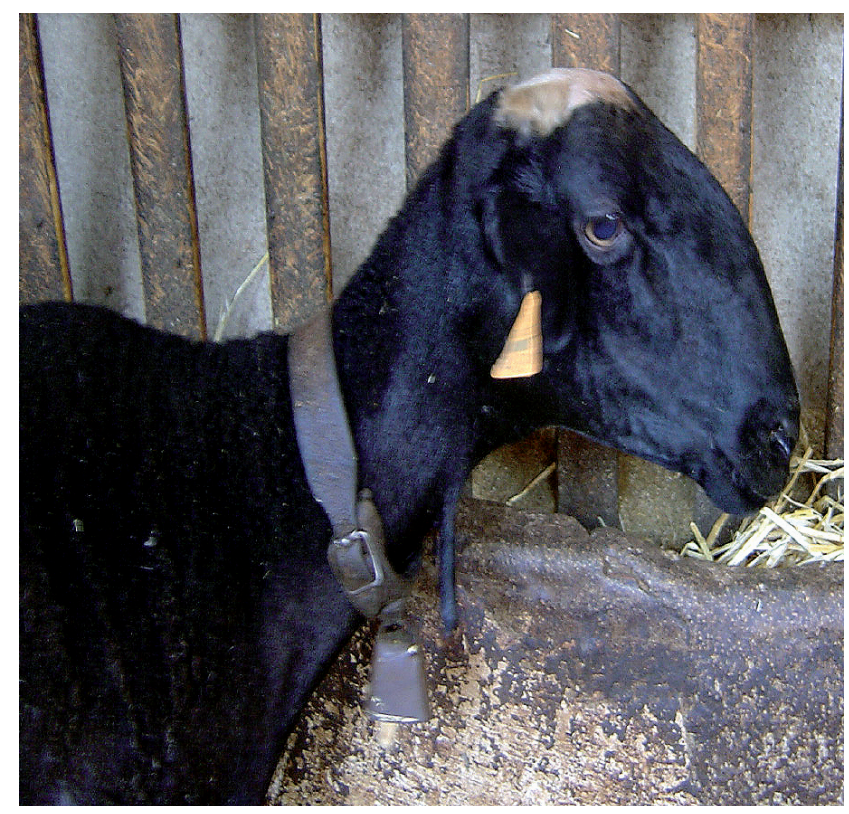

Figura 5. La capa negra en la raza ovina Maellana es poco habitual aunque periódicamente aparecen algunos ejemplares con esta coloración no admitidos por el estándar oficial de la raza. Oveja Maellana de capa negra procedente de la ganadería tarraconense de la familia Julvé de Arnes (Terra Alta) (The black layer on Maellana sheep breed is rare although some specimens appear periodically this color not supported by the official breed standard).

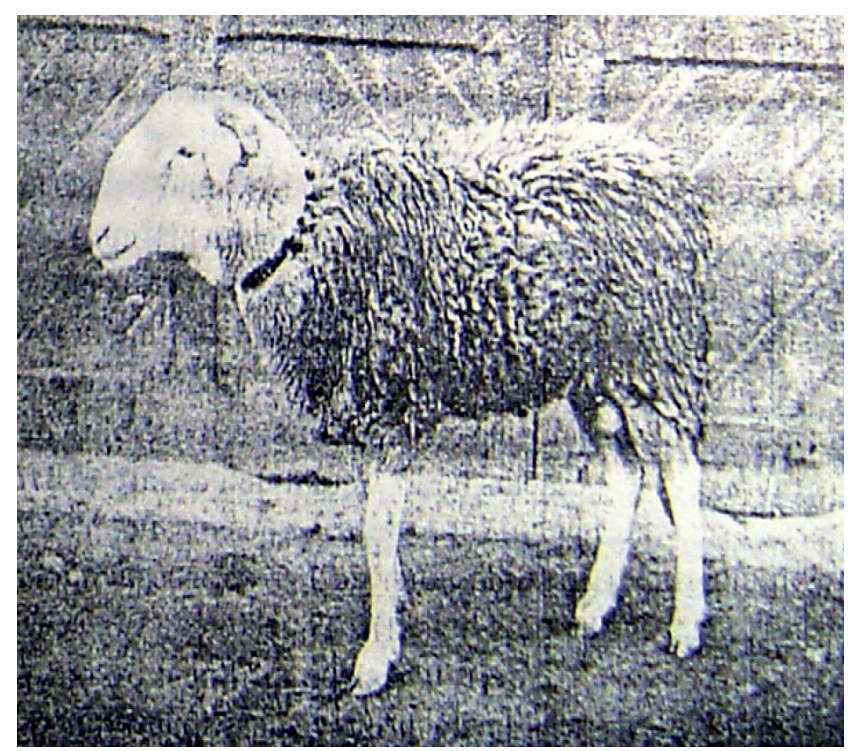

Figura 6. A finales del siglo XIX el Moltó Tarragoní estaba muy hibridado con otras razas y ya había perdido su característica cornamenta y su capa negra (1897). Archivo del Doctor Emili Giralt (In the late nineteenth century Tarragona Ram was very hybridized with other breeds and had lost its antlers characteristic and its black cloak (1897)).

Estos argumentos, nos permiten afirmar que probablemente la raza ovina Maellana y el Moltó Tarragoní, formaron parte, en el pasado, de una misma agrupación racial que con el tiempo acabó diferenciándose en dos razas independientes.

Considerando esta posibilidad, el territorio histórico de la raza tarraconense podría considerarse una extensión natural del de la raza Maellana, en el que se conservó la forma más primitiva y genuina.

\section{ORIGEN, TRADICIÓN E HISTORIA}

Según la clasificación de los ovinos españoles establecida por Sánchez et al. (1979), el Moltó Tarragoní quedaría encuadrado dentro del troco entrefino. Su representante ancestral, tradicionalmente ha sido identificado con el Ovis aries ligeriensis, tipo primitivo de Europa Central que llegó a Francia y a través de los Pirineos penetró en la península ibérica.

Forcada Miranda (1984) indica que en aquellas razas en las que coinciden las capas blanca y negra, el color negro fue anterior al blanco, para posteriormente quedar circunscrito a determinadas zonas montañosas y pobres. En aquellas razas en las que actualmente conviven las capas blancas y las negras, la variedad negra parece representar la mayor pureza racial y en algunos casos la original de la raza.

Emili Giralt i Raventós incluye a la raza tarraconense dentro del conocido como tipo pirenaico, descrito por Sansón (1910).

En el distrito francés del Bearn y en el País Vasco francés existe una raza ovina denominada Vasco-Bearnesa que guarda cierto parecido con el Moltó Tarragoní.

El Moltó Tarragoní aparece ya documentado con esta denominación en el siglo XVII. Para ser más exactos, en el año 1687 la Universidad de Vilafranca del Penedés y su Iglesia parroquial establecieron una concordia en la que se repartían ciertos privilegios y ya se mencionaba la raza (Bosch et al., 1992).

Es pactat y concordat entra las matexas parts y per evitar lo litigi en quant a la taxa del número dels moltons que la dita reverent Comunitat podía fer péxer y pasturar per lo terme y territorio de dita vila. Per co, la dita Universitat de Vilafrancha de Panadès, ab thenor del present capitol, convé y promet a dita reverent Comunitat de Preveres de la iglesia Parrochial de la matexa vila, que puga y le sie lícit y permès tenir un remat de moltons negres, dits tarragonins, de número de cent y trenta caps dins lo dit terme y territori, y de fer depéxer y pasturar las erbas de las terras, així cultivas, hermas, boscosa y montayonas de aquel, per totas aquellas parts y partidas que lo bestiar propri de la matexa Universitat, e, o del asegurador de les Carniserias Públicas y comunas de la matexa Universitat. Ab expresa declaratió que los dits cent y trenta caps de moltons tíngan de anar asenyalats ab lo señal o armas que usa dita reverent Comunitat, que són dos ulls.

\section{TIPOS DE PRODUCCIÓN}

El Moltó Tarragoní estaba especializado en la producción de carne de excelente calidad y, a diferencia de otras poblaciones ovinas, su lana era de muy baja calidad. La presencia de lana era considerada un elemento no deseable y que acababa repercutiendo de forma negativa en la calidad de la carne. Ocupándose de este asunto Rovira (1884) indica:

los carneros dan la bondad de su carne en razón inversa de la bondad de lana; un carnero de buena lana dará carne malísima y uno de lana mala carne buenísima. 


\section{CAUSAS DE SU EXTINCIÓN}

La importancia de la carne de carnero en la dieta catalana ha sido muy importante. Francisco de Zamora señalaba, a finales del siglo XVIII, que la gente acomodada de Barcelona consumía mucha carne, siempre de carnero, mientras que los pobres consumían poca carne y generalmente de oveja, caprino o vaca (Gras et al., 2000).

A finales del siglo XIX, en plena crisis finisecular, se producen una serie de cambios socioeconómicos $\mathrm{y}$ alimentarios que afectaron de una manera determinante al devenir y conservación de esta antigua raza. Dentro de este contexto de crisis, se observa un cambio de tendencia en el consumo de carne de carnero, prefiriéndose en este periodo animales de menor tamaño que los que producía la raza tarraconense. Contribuyeron a este fin los intereses de los carniceros, quienes pagaban impuestos a razón del peso y no del número de animales sacrificados. Este hecho propició que se prefiriese sacrificar un mayor número de animales de menor talla que reportaban mayor números de menudos, cabezas e hígados, alimentos de gran consumo en aquel contexto histórico. En segundo lugar también intervinieron los intereses de los consumidores, quienes no siempre valoraban la bondad i calidad de la carne del Moltó Tarragoní. March (1897), señala que las amas de casa preferían comprar una docena de costillas de esta carne para el uso diario que un menor número de las mismas pero de mayor peso, ya que estas buscaban un precio económico y un reparto fácil.

Este cambio en los hábitos alimenticios locales propició que en pocas décadas la raza desapareciese de la mayoría de explotaciones ganaderas. Los ganaderos cruzaban sus cabañas con sementales de otras agrupaciones raciales de procedencia aragonesa, manchega o levantina que acabaron sustituyendo la población original (figura 6).

En 1866 algunos propietarios de Sant Sadurní d'Anoia, cansados de comer carne de baja calidad se juntaron en sociedad, para defender la calidad de las carnes que consumían (Rovira, 1884).

Cansados los propietarios de esta villa de comer carne mala se juntaron en sociedad en el año 1866 i desde esta fecha se come aquí carnero tarraconense y es fama por nadie desmentida que nosotros comemos la mejor carne de España $i$ esto es lo que tendría Barcelona [...].

Existieron algunos intentos proteccionistas hacia la carne de Moltó Tarragoní. En 1884 se encomendó al ayuntamiento de Barcelona que una comisión estudiase el modo de corregir los elevados precios que gravaban algunos artículos de primera necesidad y que tenía que permitir que la raza se recuperara, pero dichos intentos no prosperaron.

Existieron otras causas que también contribuyeron a la desaparición del Moltó Tarragoní. Con la llegada del ferrocarril a Cataluña y la consecuente mejora de las comunicaciones, se incrementó el tránsito de mercancías, y la entrada de ganados con destino al abastecimiento de la ciudad de Barcelona.

La expansión de un cultivo plurianual, como la viña, impedía el aprovechamiento de las rastrojeras y también contribuyó a reducir la actividad ganadera. Durante el siglo XVIII, gracias a la demanda de aguardiente de los países del norte de Europa y al desarrollo del mercado americano, algunas comarcas catalanas empezaron a especializarse de forma exclusiva en la producción de vino. Las consecuencias de este cambio de actividad también repercutieron de forma importante en la población ovina local. A principios del siglo $X X$, la antigua raza tarraconense desaparece para siempre.

\section{BIBLIOGRAFÍA}

Barallat, H. 1884. Excursió a Sant Pere de Riudevitlles, Sant Quintí de Mediona i Mediona. Memòries de la Associació Catalanista d'Excursions Científiques, XII: 51 1-537.

Gras, M. i Pérez, M.A. 2000. Els tractats d'agricultura i alimentació pagesa a l'època moderna. Estudis d'Història Agrària, 13: 63-106.

Bosch, J.M.; Coll, M.C.; Vallés, J. i Vidal, J. 1992. El Llibre Verd de Vilafranca. Vol. II. Fundació Noguera. Barcelona.

FAO. 2010. Extint breeds list. http://www.fao.org/dad-is/ (02/02/2014).

Forcada, F. 1984. Estudio etnológico y productivo de la agrupación ovina roya bilbilitana. Tesis doctoral. Universidad de Zaragoza.

Giralt, M. i Giralt, O. 2008. Fonts documentals referents al Moltó Tarragoní recopilades per Emili Giralt i Raventós. Estudis d'història agrària, 21: 79-88.

Hall, S.J.G. and Ruane, J. 1993. Livestock breeds and their conservation: A global overview. Conserv Biol, 7: 815-825.

March. 1897. Duas cartas. L'Art del Pagès, XIV: 113-117.

Rovira, P. 1884. Moltó Tarragoní. L'Art del Pagès, VIII: 207-209.

Scherf, B. 2000. World watch list for Domestic Animal Diversity. FAO. Roma. Third edition. http://www.fao.org/docrep/009/x8750e/ x8750e00.htm (02/02/2014).

Rischkowsky, B. and Pilling, D. 2010. La situación de los recursos zoogenéticos mundiales para la alimentación y la agricultura. FAO. Roma.

Rossell, P.M. 1931. Les races animals relacionades amb l'etnologia de Catalunya. Agricultura i Ramaderia, 14: 114-116.

Sánchez, A. y Sánchez, M.C. 1979. Razas ovinas españolas. Publicaciones de extensión agraria. Madrid.

Virgili. 1892. Provincia de Tarragona. La ganadería en España: avance sobre la riqueza pecuaria en 1891. Junta Consultiva Agronómica. Madrid. 
